\title{
Performance Analysis of ASK and PSK Modulation Based FSO System Using Coupler-Based Delay Line Filter under Various Weather Conditions
}

\author{
Mohd Ashraf, Gaurav Baranwal, Dinesh Prasad, Saima Idris, Mirza Tariq Beg \\ Department of Electronics and Communication, F/O Engineering and Technology, Jamia Millia Islamia, Delhi, India \\ Email: mohd.ashraf305@gmail.com,gauravbaranwa191@gmail.com,dprasad@jmi.ac.in, saimaidris05@gmail.com, \\ mtbeg@jmi.ac.in
}

How to cite this paper: Ashraf, M., Baranwal, G., Prasad, D., Idris, S. and Beg, M.T. (2018) Performance Analysis of ASK and PSK Modulation Based FSO System Using Coupler-Based Delay Line Filter under Various Weather Conditions. Optics and Photonics Journal, 8, 277-287.

https://doi.org/10.4236/opj.2018.88023

Received: July 18, 2018

Accepted: August 24, 2018

Published: August 27, 2018

Copyright $\odot 2018$ by authors and Scientific Research Publishing Inc. This work is licensed under the Creative Commons Attribution International License (CC BY 4.0).

http://creativecommons.org/licenses/by/4.0/

\begin{abstract}
Free space optical communication (FSO) proves to be very effective and efficient technology for wireless communication. This work basically deals with the designing of FSO systems. Further, a comparative study has been made to ascertain which modulation technique proves better for communication. This paper investigates the performance of ASK and PSK modulation based FSO system by varying different FSO parameters under several conditions including haze, rain, mist and fog. Finally, simulation results are analysed and discussed.
\end{abstract}

\section{Keywords}

FSO, Bit Error Rate (BER), Amplitude Shift Keying (ASK), Phase Shift Keying (PSK), Attenuation

\section{Introduction}

Nowadays, a very high-quality service demand for multimedia services is increasing drastically. Optical fibres have been used for so many years to fulfil this demand. But the shortcoming of these fibres is that it is not suitable for remote areas deployment and enhances the cost of the system. So, an alternative approach to meet the desired requirements is free space optics (FSO) technology. FSO uses optical wireless links to offer point to point communication in outdoor terrestrial regions with clear line of sight (LOS). FSO system has an optical transceiver unit which has a bi-directional functionality. Each unit involves an 
optical source, telescope which transmits light through free space, which behaves as a communication channel in it, to another telescope and receives the information [1].

Advantages of FSO include high reuse factor, robustness, easily deployable, license free band, no signal interference. FSO system is used for various applications, such as in LAN networks for campus connectivity with Gigabyte Ethernet speeds, in metropolitan area network, spacecraft communication, video surveillance, back-haul for cellular networks like $5 \mathrm{G}$ etc. [2].

Unfortunately, FSO links are prone to inhomogeneities produced by the temperature and pressure in the atmosphere. This causes atmospheric turbulence effect. This results fluctuations in the received signal, called as scintillation effect or turbulence-induced fading. This is analogous to fading in RF system. Due to this, FSO suffers with the drawback of less reliable link connectivity mainly for distances more than 1000 metre and is sensitive to various weather conditions [3] [4] [5].

\section{System Architecture}

\subsection{ASK Modulation Technique}

The block diagram of the proposed FSO system using ASK modulation technique is shown in Figure 1. It can be divided into 4 stages. First stage is transmitter which contains an optical source, bit sequence generator, pulse generator and AM modulator. Here pulse generator encodes the digital data of bit sequence generator and its output modulated the carrier signal of optical source using amplitude modulator. Second stage is delay line filter. It is utilised to compensate the dispersion effects which, in turn, reduces the effects caused by atmospheric turbulence, fading etc. the coupling coefficients of the coupler present in this filter can change the dispersion slope. Hence, dispersion and dispersion slope value of the filter can be designed accordingly [6] [7]. The third stage is FSO channel as described earlier. The final stage is the receiver where the optical detector detects the signal and converts it into the electrical signal.

\subsection{PSK Modulation Technique}

The block diagram of the proposed FSO system using PSK modulation technique is shown in Figure 2. It consists of 4 stages. First stage is transmitter stage.

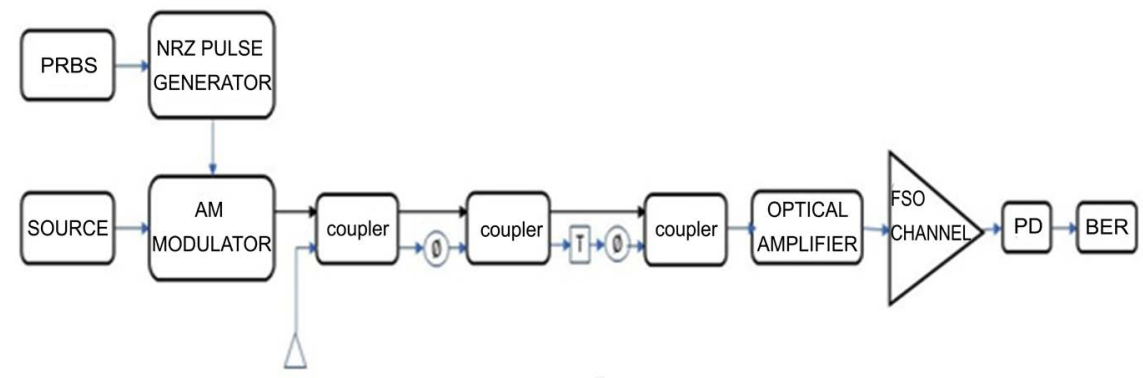

Figure 1. Configuration of ASK modulation technique setup. 


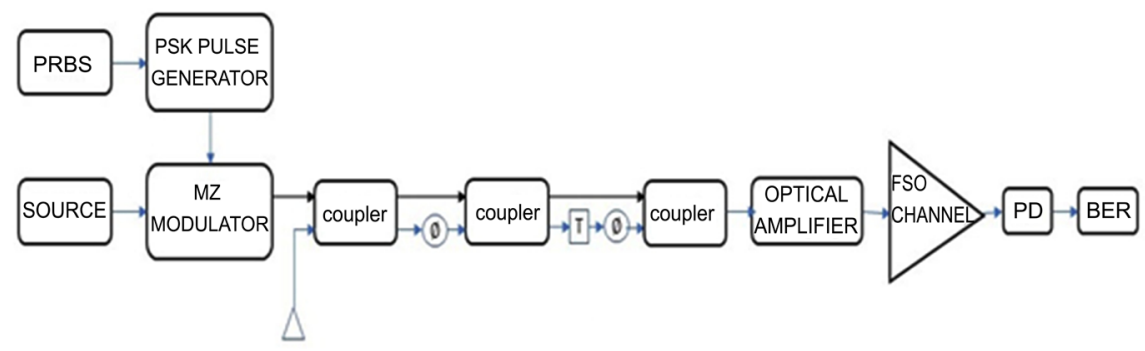

Figure 2. Block diagram of the proposed PSK modulated FSO system.

In this PSK pulse generator encodes the digital data of bit sequence generator. Encoded data modulates the optical carrier signal using phase modulator. Second stage is delay line filter used to compensate dispersion effects and reduce the turbulence and fading caused by it. The stage is FSO channel where atmosphere acts as the channel. Fourth stage is the receiver stage for the detection of the optical signal and converting it into an electrical signal.

\section{Simulation Setup}

\subsection{ASK Modulated FSO Design Simulation}

A design of ASK modulation FSO system was simulated and modelled for performance analysis under various weather condition using OptiSystem software shown in Figure 3. The optical transmitter consists of Pseudo-Random Binary Sequence (PRBS) generator representing data bits of bit rate 10Gbits/sec. The output of this is encoded with Non-Return to Zero (NRZ) pulse generator. A continuous wave laser of $60 \mathrm{dBm}$ and wavelength $1550 \mathrm{~nm}$ is modulated by the encoded bits using optical amplitude modulator. The output of the modulator is the optical signal which is fed into the delay line filter using $\mathrm{x}$-couplers. The first coupler decomposes the input signal into two, depending on the coupling coefficient. One of the split signals is phase shifted and the other one is kept as it is. Then they are recombined at the second coupler. These are again split and time delayed and phase shifted. Finally, at the third coupler, they are combined to receive the compensated signal. The free space optics medium (FSO channel) with different range and attenuations has been considered and analysed. The optical receiver consists of PIN photodiode and a 3R-regenerator. The PIN photodiode converts received optical signal into the corresponding electrical signal [8]. The $3 \mathrm{R}$-regenerator obta in the original bit sequence. The oscilloscope visualizers and bit error rate (BER) analyzer are used to analyse the results obtained.

\subsection{PSK Modulated FSO Design Simulation}

A design of PSK modulated FSO system was simulated and modelled for performance analysis under various weather conditions using Opti-System software. Figure 4 shows the simulation structure of proposed PSK modulated FSK system. In the optical transmitter, data bit of $10 \mathrm{Gbits} / \mathrm{sec}$ is generated using PRBS generator which is then encoded with the help of PSK pulse generator. $\mathrm{CW}$ laser of $60 \mathrm{dBm}$ and $1550 \mathrm{~nm}$ wavelength was used to generate optical 


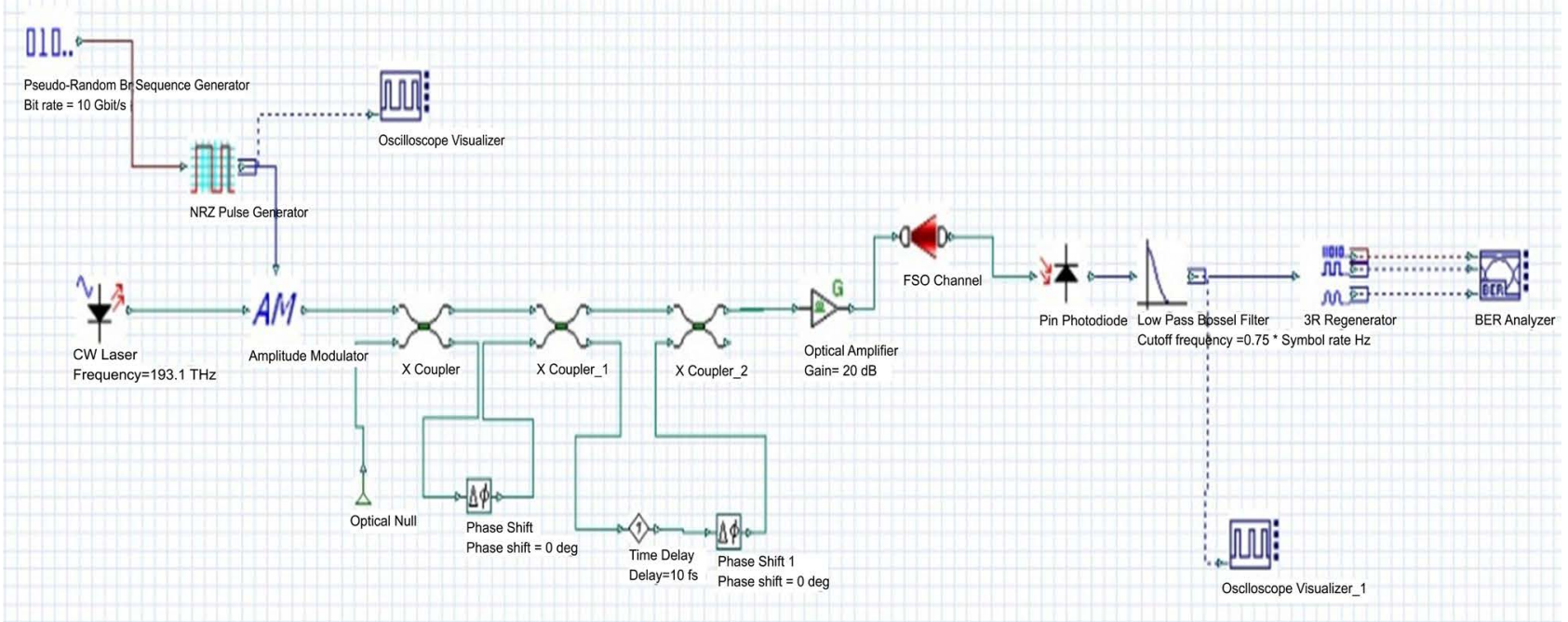

Figure 3. Simulation structure for ASK modulated FSO system.

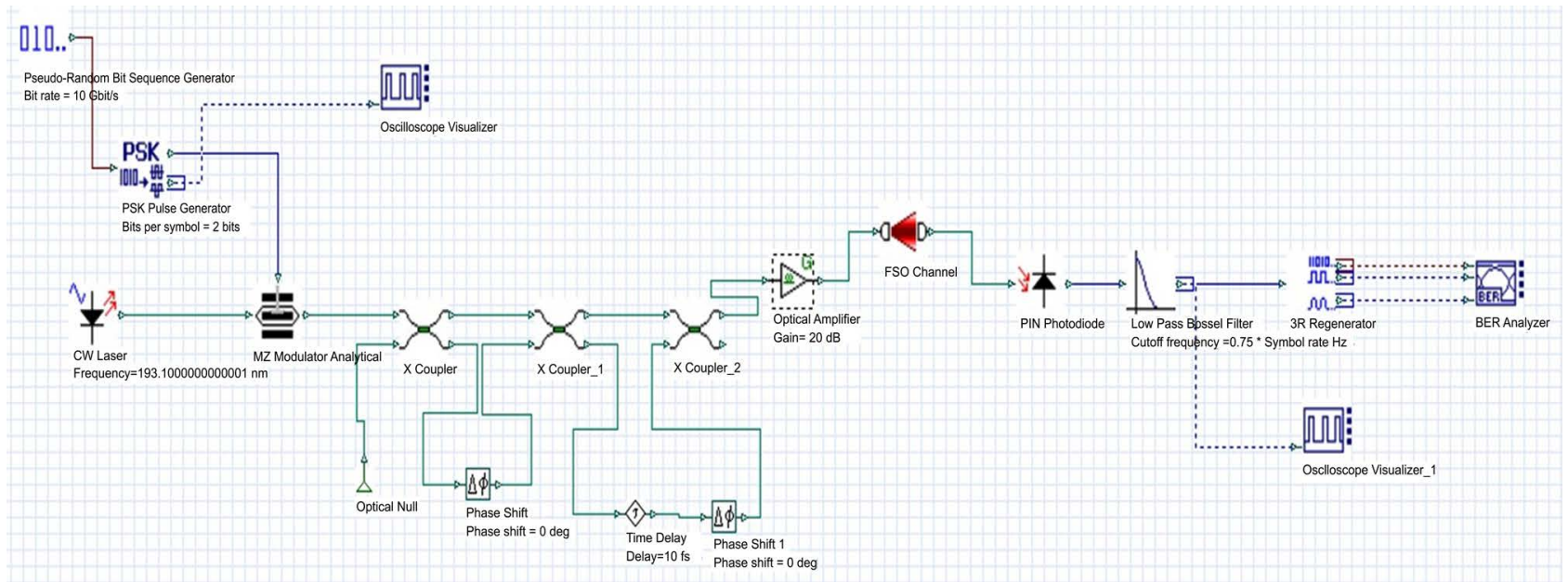

Figure 4. Simulation structure for PSK modulated FSK system.

carrier signal output of PSK pulse generator modulates the optical carrier signal phase resulting in the PSK modulated signal. Modulated signal is passed through the delay line filter to improve the performance of the FSO system as explained earlier. Compensated signal is passed through the FSO channel. The performance is analysed by taking different ranges and attenuation values of the FSO channel corresponding to different weather conditions. The optical receiver has PIN photodiode and a 3R-regenerator to obtained original bit sequence in electrical signal form. Finally, the performance is analysed with the help of oscilloscope visualizers and BER analyzer.

\subsection{FSO System Parameters under Various Atmospheric Conditions}

The author in [9] [10] described the effects of several weathers conditions for different atmospheric turbulences including rain, fog, haze, etc on FSO systems 
and given the maximum visibility range and attenuation present for such weather conditions. Table 1 contain FSO system parameters under various conditions. Free space optical system parameters for various weather conditions like haze, rain-mist, snow and medium Fog are included in Tables 2-5 respectively.

Table 1. Free space optical system parameters under various atmospheric conditions.

\begin{tabular}{cc}
\hline ATMOSPHERIC CONDITION & ATTENUATION $(\mathrm{dB} / \mathrm{km})$ \\
\hline HAZE & $10.94-20.68$ \\
Rain & $6.0-30$ \\
Mist & $28.56-31.45$ \\
Snow & 40 \\
Mid-Fog & 70 \\
\hline
\end{tabular}

Table 2. Result analysis for Haze condition.

\begin{tabular}{cccccc}
\hline Work & $\begin{array}{c}\text { Attenuation } \\
(\mathrm{dB} / \mathrm{km})\end{array}$ & LOS (metre) & Q-factor & BER & $\begin{array}{c}\text { Bit Rate } \\
(\mathrm{Gbps})\end{array}$ \\
\hline Ref [11] (ASK) & 20 & $100 \mathrm{~m}$ & 3.86 & $5.03 \mathrm{e}-005$ & 10 \\
Ref [12] & 20 & $800 \mathrm{~m}$ & 21.08 & - & 2.5 \\
Ref [13] & 20 & $500 \mathrm{~m}$ & 45.96 & 0 & 10 \\
ASK DLF (proposed) & 20 & $3500 \mathrm{~m}$ & 56.5069 & 0 & 10 \\
PSK DLF (proposed) & 20 & $3500 \mathrm{~m}$ & 57.0486 & 0 & 10 \\
\hline
\end{tabular}

Table 3. Result analysis for rain and mist condition.

\begin{tabular}{cccccc}
\hline Work & $\begin{array}{c}\text { Attenuation } \\
(\mathrm{dB} / \mathrm{km})\end{array}$ & LOS (metre) & Q-factor & BER & $\begin{array}{c}\text { Bit Rate } \\
(\mathrm{Gbps})\end{array}$ \\
\hline Ref [11] (ASK) & 30 & 100 & 3.86079 & $5.025 \mathrm{e}-005$ & 2.5 \\
Ref [12] & 30 & 800 & 9.62 & - & 2.5 \\
ASK DLF (proposed) & 30 & 2500 & 31.4472 & $2.292 \mathrm{e}-217$ & 10 \\
PSK DLF (proposed) & 30 & 2500 & 34.0397 & $2.855 \mathrm{e}-254$ & 10 \\
\hline
\end{tabular}

Table 4. Result analysis for snow condition.

\begin{tabular}{cccccc}
\hline Work & $\begin{array}{c}\text { Attenuation } \\
(\mathrm{dB} / \mathrm{km})\end{array}$ & LOS (metre) & Q-factor & BER & $\begin{array}{c}\text { Bit Rate } \\
(\mathrm{Gbps})\end{array}$ \\
\hline Ref [14] & 40 & - & 30.2722 & $9.174 \mathrm{e}-202$ & 2.5 \\
ASK DLF (proposed) & 40 & 1900 & 44.994 & 0 & 10 \\
PSK DLF (proposed) & 40 & 1900 & 46.139 & 0 & 10
\end{tabular}

Table 5. Result analysis for medium Fog condition.

\begin{tabular}{cccccc}
\hline Work & $\begin{array}{c}\text { Attenuation } \\
(\mathrm{dB} / \mathrm{km})\end{array}$ & LOS (metre) & Q-factor & BER & $\begin{array}{c}\text { Bit Rate } \\
(\mathrm{Gbps})\end{array}$ \\
\hline Ref [13] & 70 & 500 & 2.934 & 0.00160863 & 10 \\
Ref [14] & 70 & - & 2.59 & 0.00153508 & 2.5 \\
ASK DLF (proposed) & 70 & 1200 & 16.1147 & $1.004 \mathrm{e}-058$ & 10 \\
PSK DLF (proposed) & 70 & 1200 & 17.5354 & $3.841 \mathrm{e}-069$ & 10 \\
\hline
\end{tabular}


Proposed ASK delay line filter and PSK delay line filter under haze condition have $3500 \mathrm{~m}$ line of sight compared to previous published work and having a high quality factor of 56.5069 and 57.0486 respectively.

Proposed ASK delay line filter and PSK delay line filter under rain and mist condition have $2500 \mathrm{~m}$ line of sight compared to previous published work and having a high quality factor of 31.4472 and 34.0397 respectively.

Proposed ASK delay line filter and PSK delay line filter under snow condition have $1900 \mathrm{~m}$ line of sight compared to previous published work and having a high quality factor of 44.994 and 46.139 respectively.

Proposed ASK delay line filter and PSK delay line filter under haze condition have $1200 \mathrm{~m}$ line of sight compared to previous published work and having a high quality factor of 16.1147 and 17.5354 respectively.

Eye diagram for various weather conditions of ASK DLF and PSK DLF is mentioned in Table 6 that include maximum quality factor, minimum BER and threshold.

\section{Conclusion}

A new approach for performance analysis of ASK and PSK modulation based FSO system using coupler-based Delay Line Filter under various weather conditions

Table 6. Eye diagram for various weather conditions.

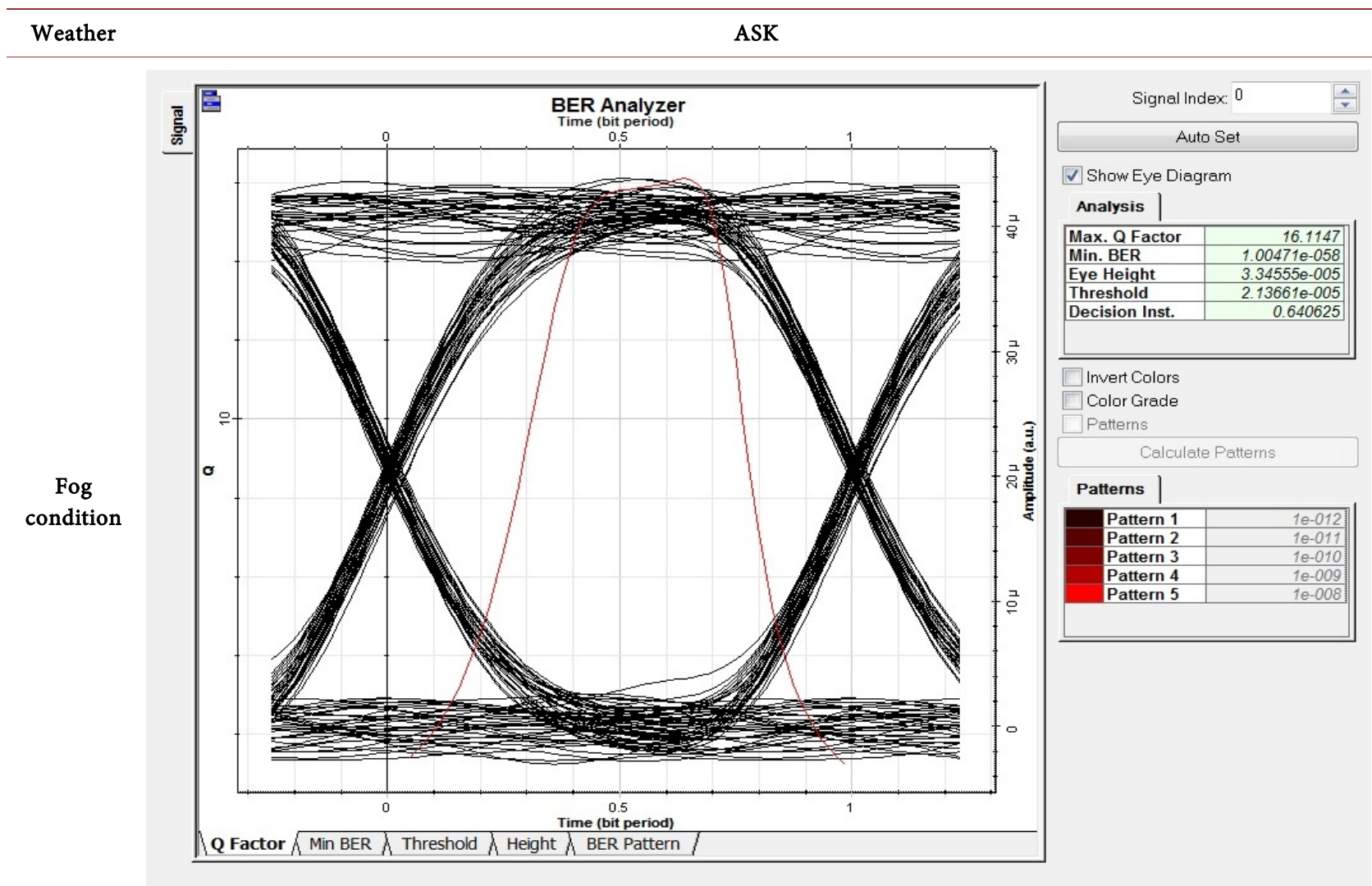

Figure 5. ASK DLF_1.2 KM_70 dB/km 10 Gbps BER. 


\section{Continued}

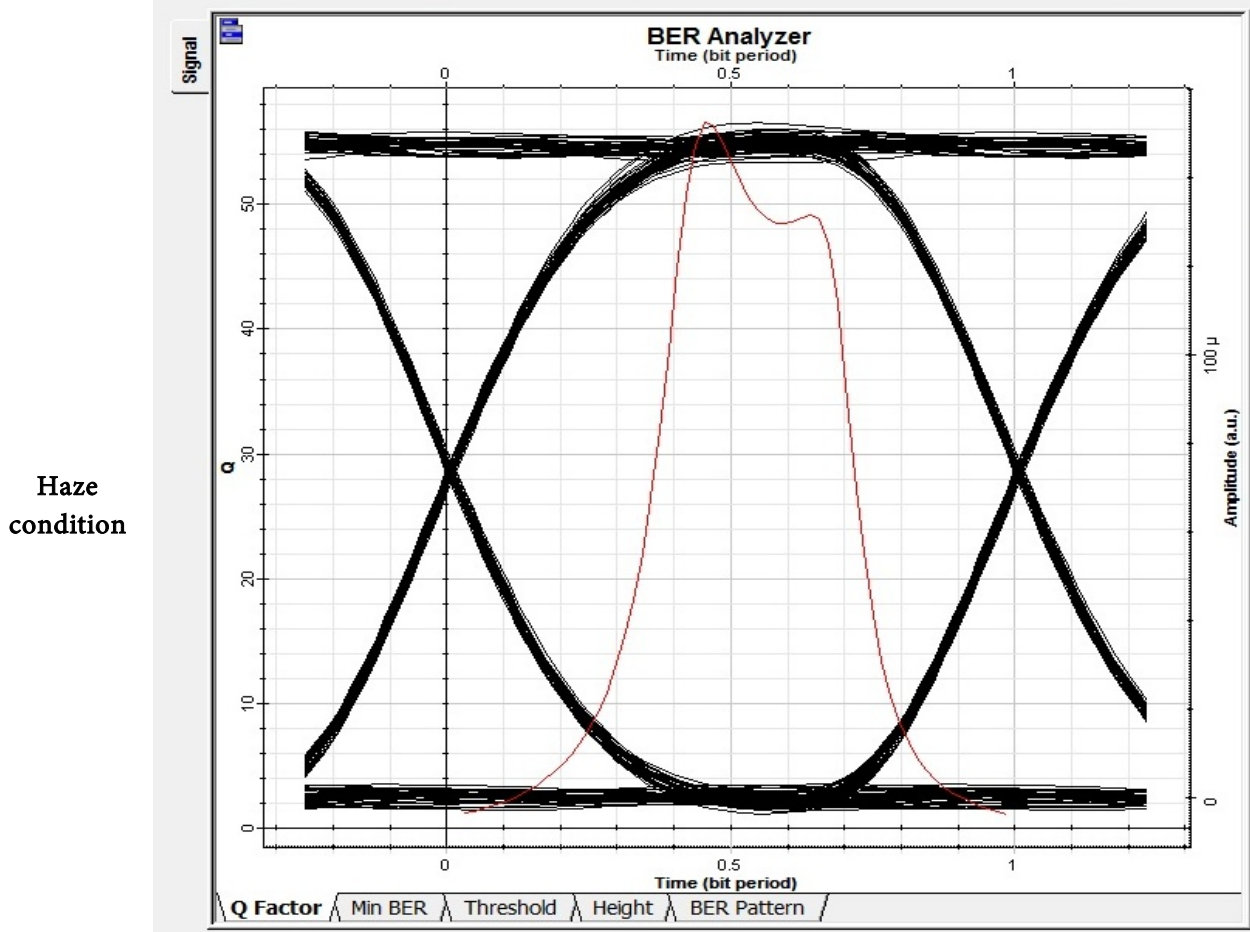

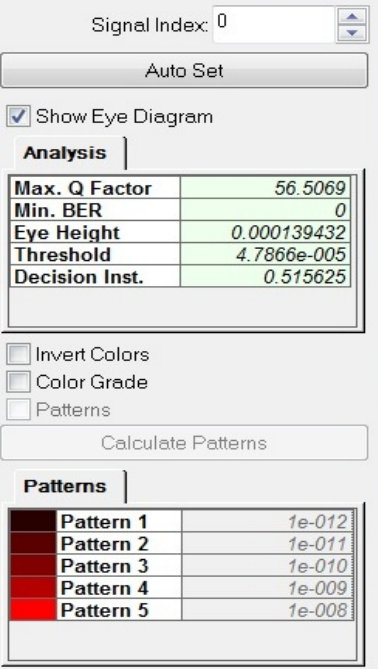

Figure 6. 10Gbps BER ASK DLF_3.5 KM_20 dB/km.
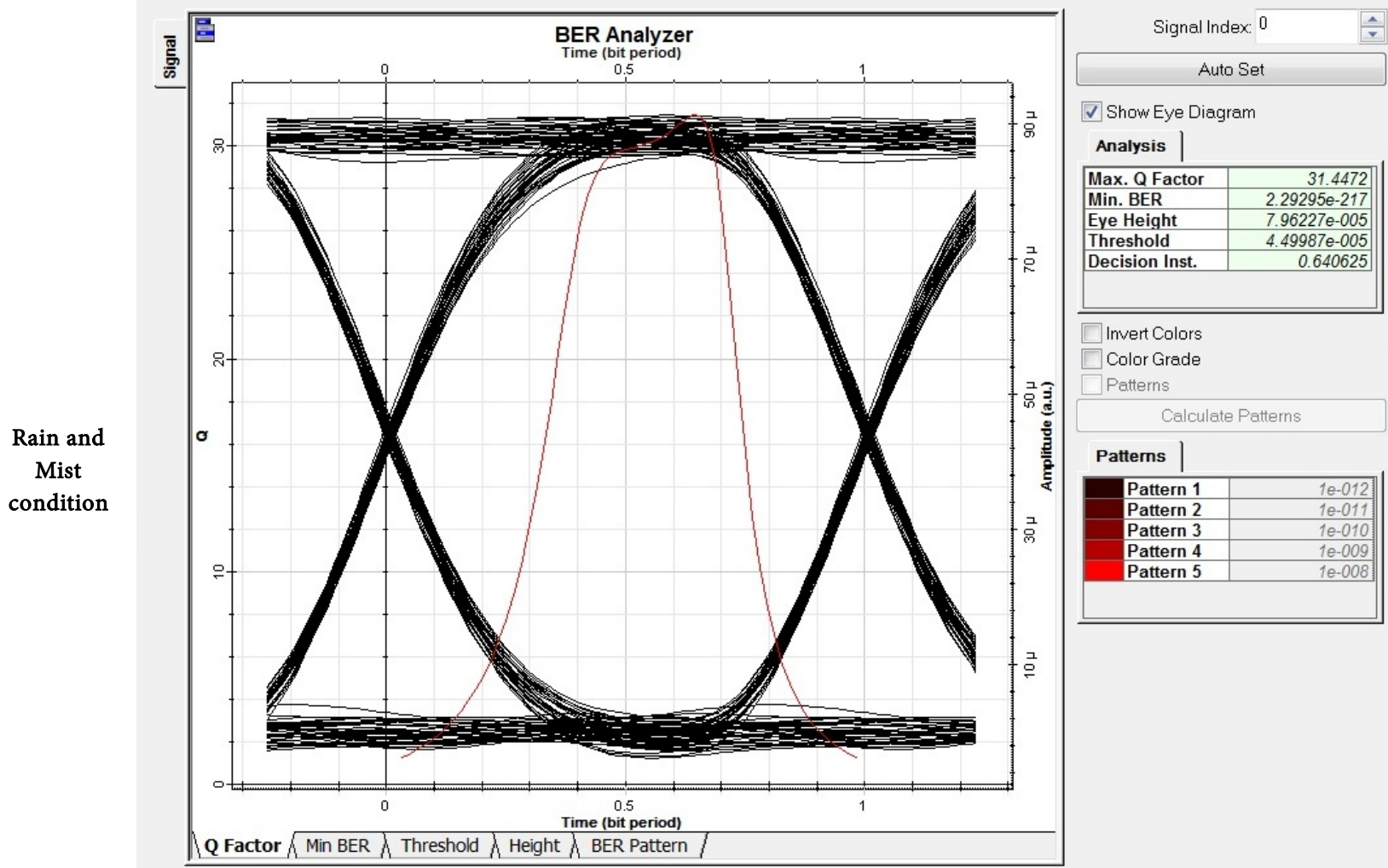

Figure 7. ASK DLF_2.5 KM_30 dB/km 10 Gbps BER. 


\section{Continued}

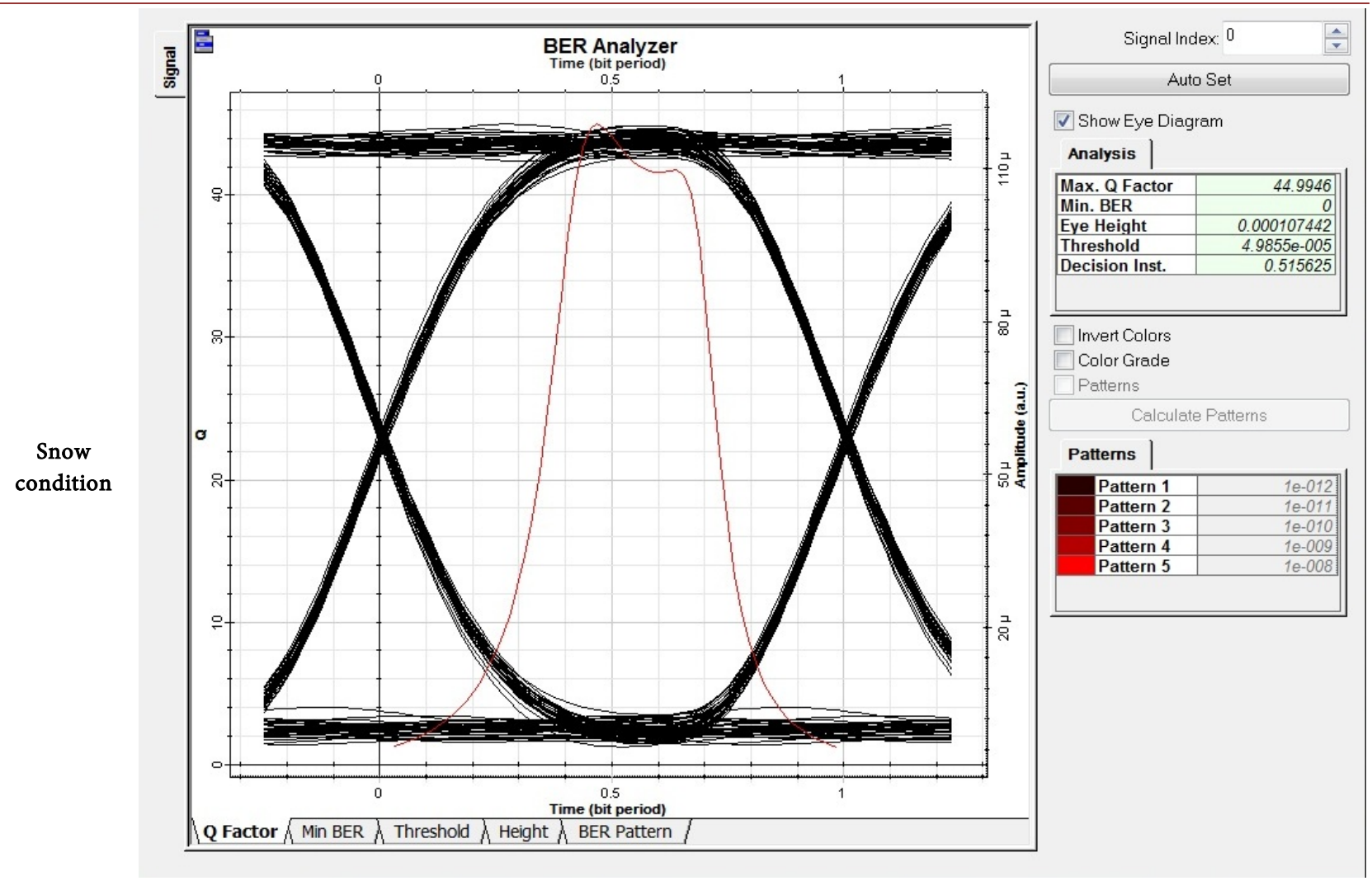

Figure 8. ASK DLF_1.9 KM_40 dB/km 10 Gbps BER.

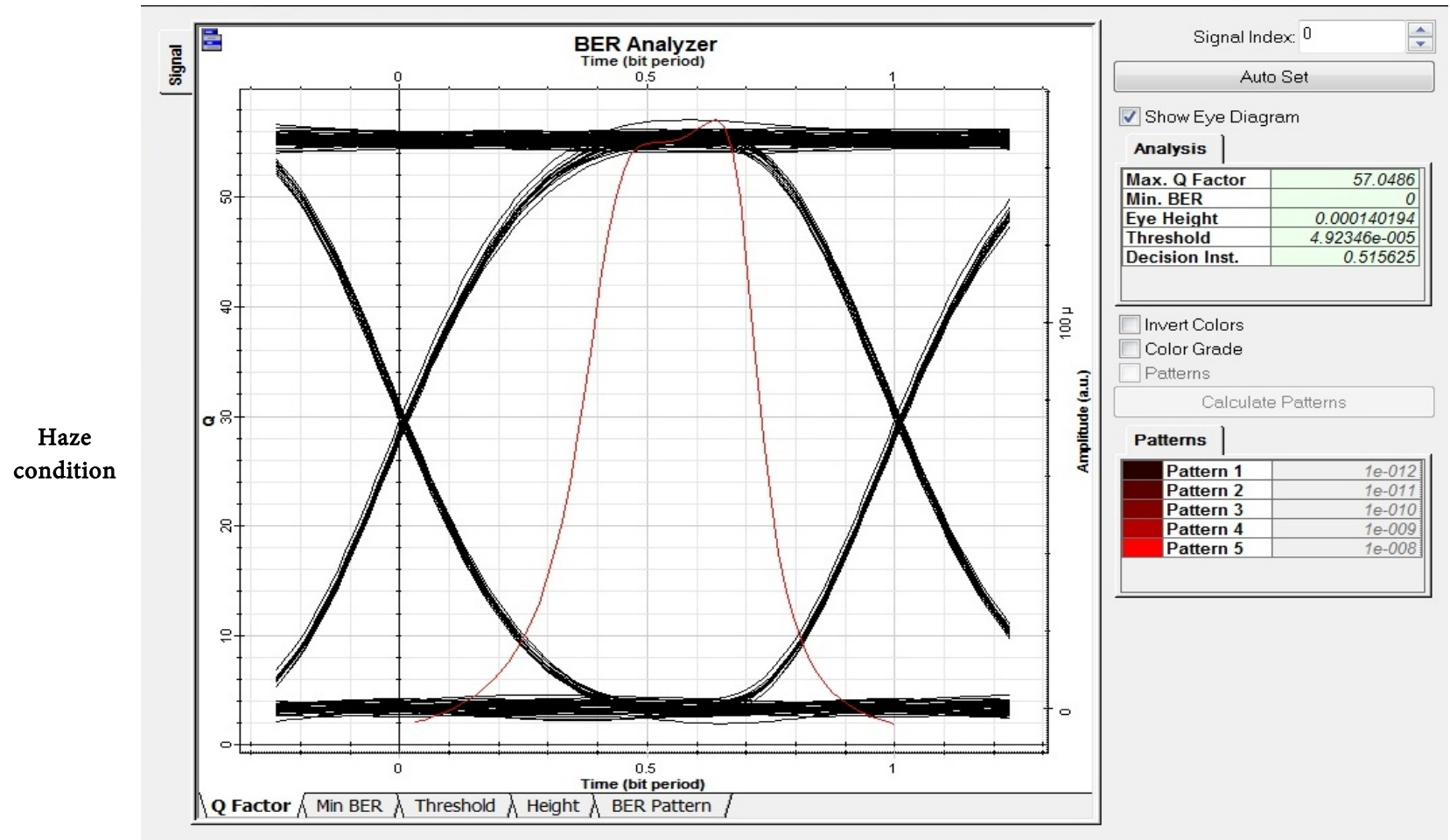

Figure 9. $10 \mathrm{Gbps}$ BER PSK DLF_3.5 KM_20 dB/km. 


\section{Continued}

Rain and

Mist

condition

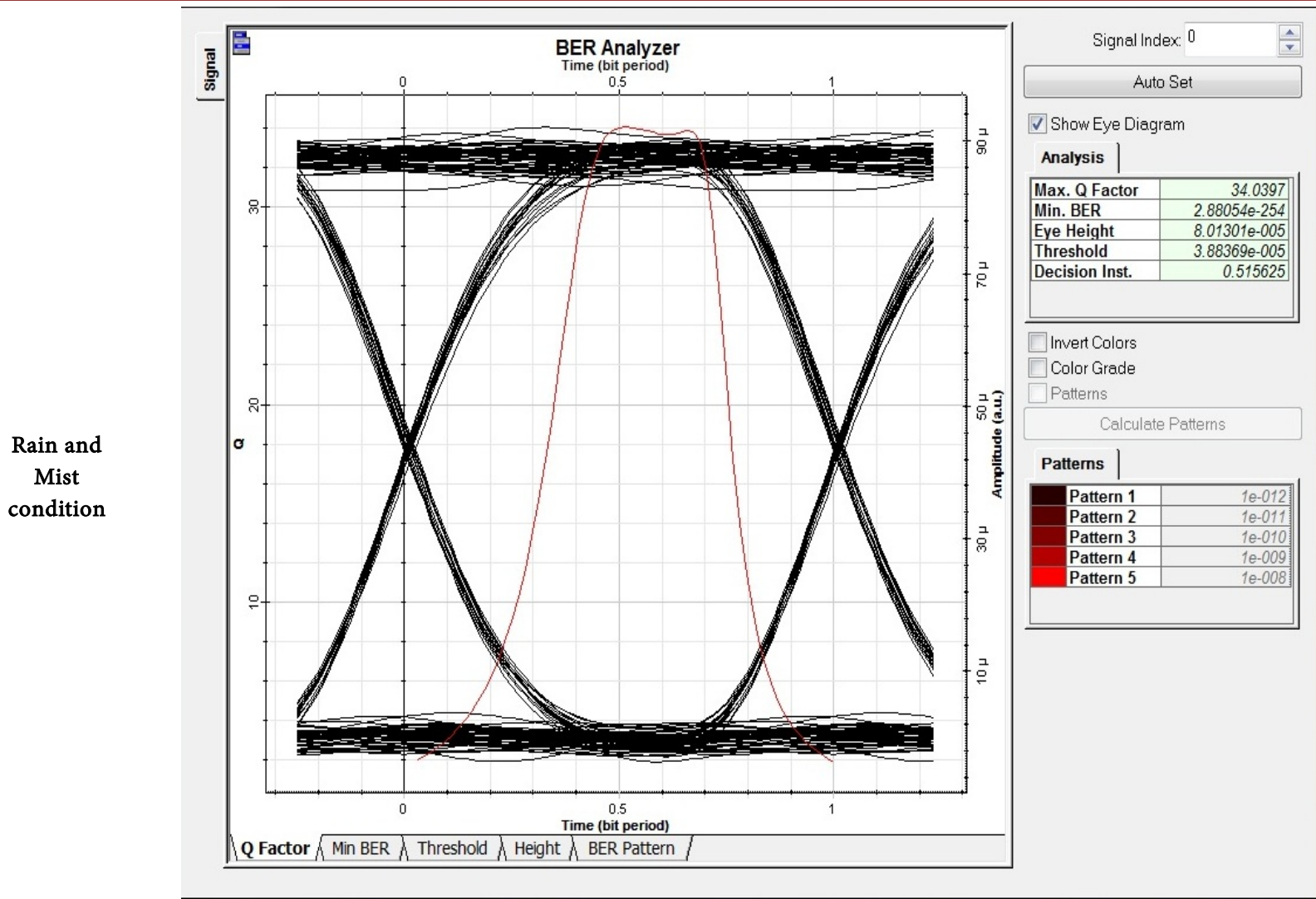

Figure 10. PSK DLF_2.5 KM_30 dB/km 10 Gbps BER.

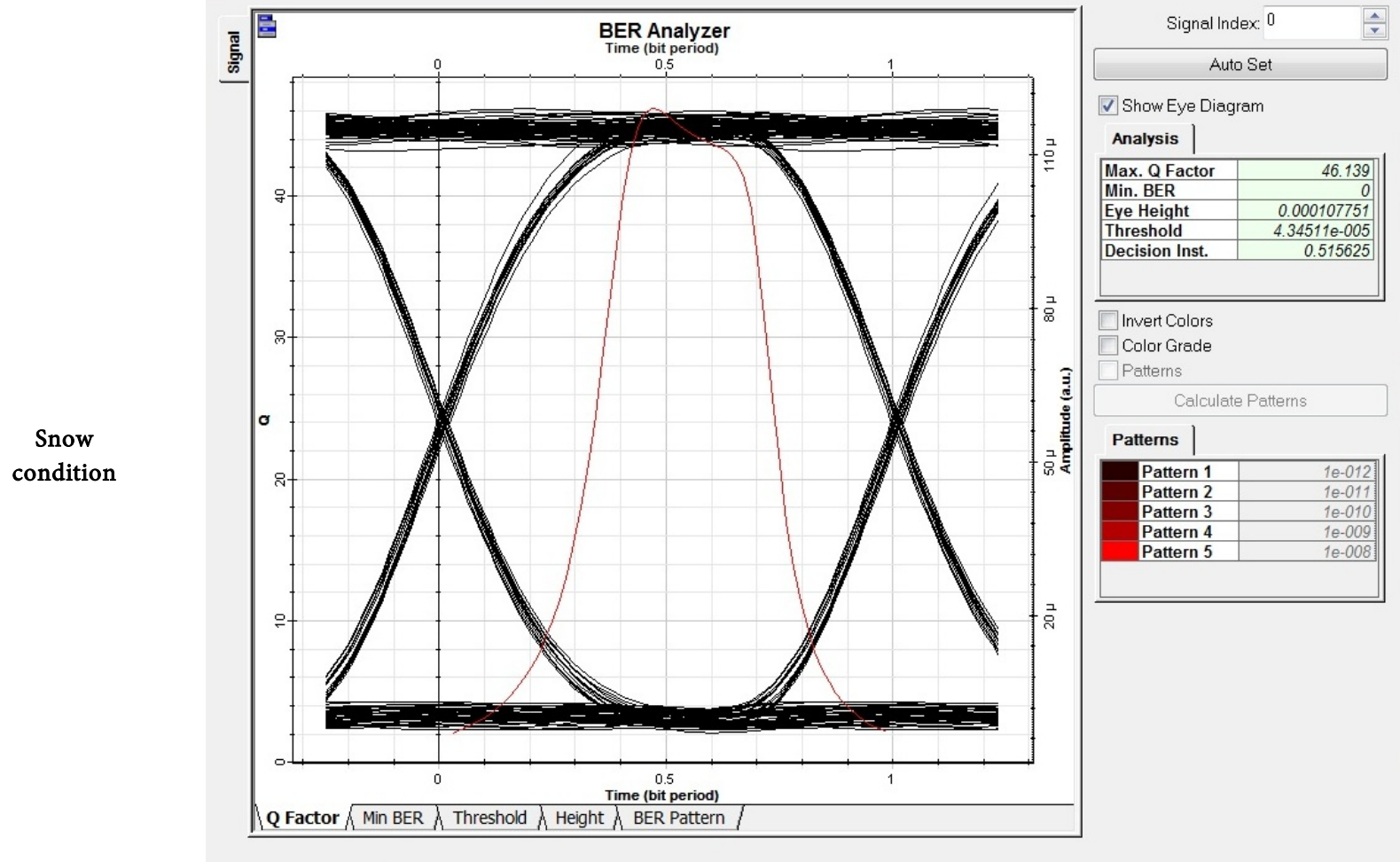

Figure 11. PSK DLF_1.9 KM_40 dB/km 10 Gbps BER. 


\section{Continued}

Fog condition
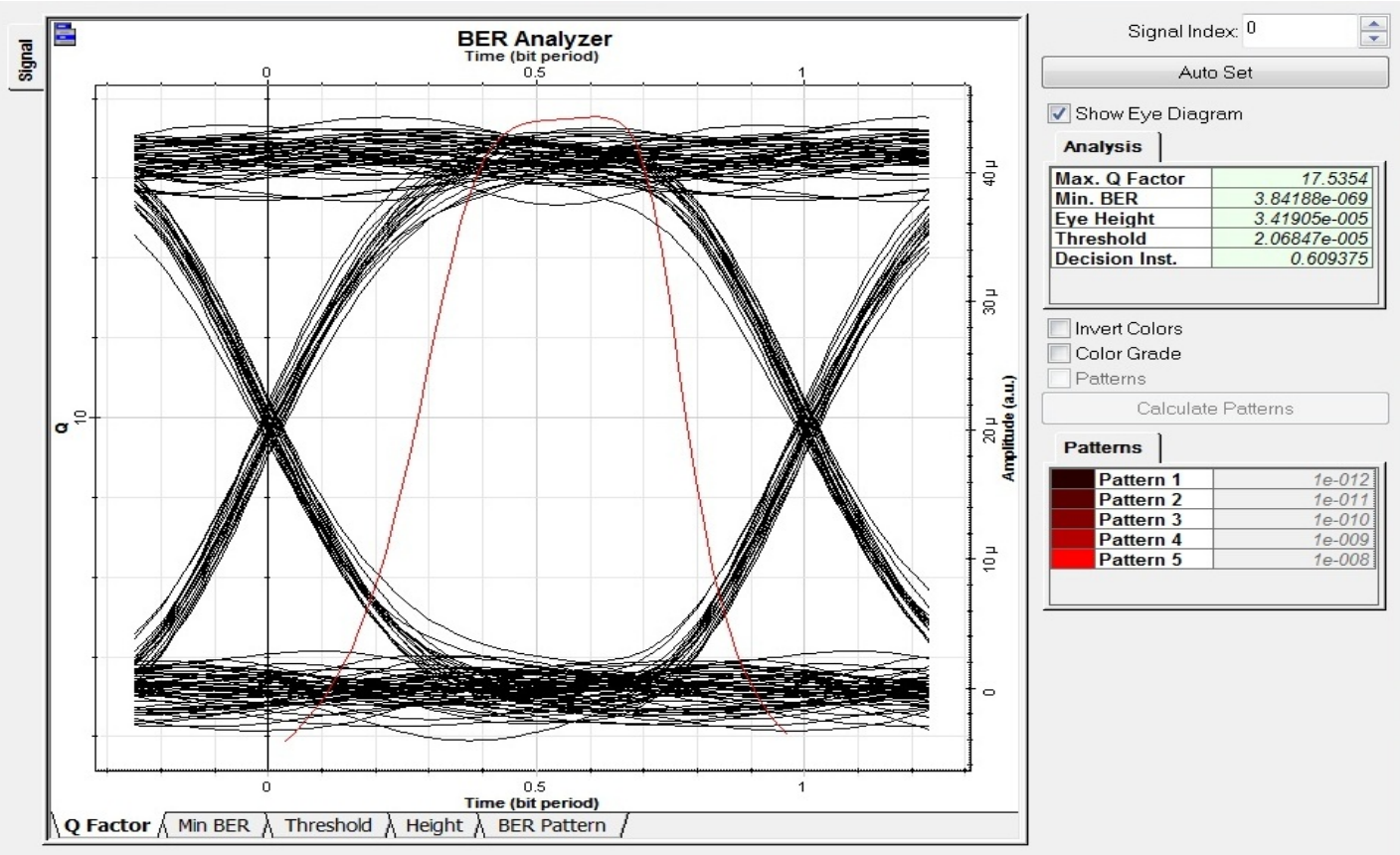

Figure 12. PSK DLF_1.2 KM_70 dB/km 10 Gbps BER.

has been presented having a feature of high Q-factor and BER is very close to zero.

\section{Conflicts of Interest}

The authors declare no conflicts of interest regarding the publication of this paper.

\section{References}

[1] Khalighi, M.A. and Uysal, M. (2014) Survey on Free Space Optical Communication: A Communication Theory Perspective. IEEE Communications Surveys \& Tutorials, 16, 2231-2258. https://doi.org/10.1109/COMST.2014.2329501

[2] Alzenad, M., Shakir, M.Z., Yanikomeroglu, H. and Alouini, M.S. (2018) FSO-Based Vertical Backhaul/Fronthaul Framework for 5G+ Wireless Networks. IEEE Communications Magazine, 56, 218-224. https://doi.org/10.1109/MCOM.2017.1600735

[3] Varotsos, G.K., Stassinakis, A.N., Nistazakis, H.E., Tsigopoulos, A.D., Peppas, K.P., Aidinis, C.J. and Tombras, G.S. (2014) Probability of Fade Estimation for FSO Links with Time Dispersion and Turbulence Modeled with the Gamma-Gamma or the I-K Distribution. Optik-International Journal of Light and Electron Optics, 125, 7191-7197. https://doi.org/10.1016/j.ijleo.2014.08.047

[4] Zhu, X.M. and Kahn, J.M. (2002) Free-Space Optical Communication through Atmospheric Turbulence Channels. IEEE Transactions on Communications, 50, 1293-1300. https://doi.org/10.1109/TCOMM.2002.800829

[5] Kong, L., Xu, W., Hanzo, L., Zhang, H. and Zhao, C. (2015) Performance of a Free-Space-Optical Relay-Assisted Hybrid RF/FSO System in Generalized \$M\$-Distributed Channels. IEEE Photonics Journal, 7, 1-19. 
https://doi.org/10.1109/JPHOT.2015.2470106

[6] Duthel, T., et al. (2006) Quasi-Analytic Synthesis of Nonrecursive Optical Delay Line Filters for Reliable Compensation of Dispersion Effects. Journal of Lightwave Technology, 24, 4403-4410. https://doi.org/10.1109/JLT.2006.881477

[7] Rahim, A., et al. (2012) Finite Impulse Response Filter Using 4-Port MMI Couplers for Residual Dispersion Compensation. Journal of Lightwave Technology, 30, 990-996. https://doi.org/10.1109/JLT.2012.2185038

[8] Ashraf, M. and Ranjan, R. (2018) Tunable Single Passband Microwave Photonic Filter Based on Direct Generation Technique. 3rd International Conference on Microwave and Photonics (ICMAP), Dhanbad, 9-11 February 2018, 1-2. https://doi.org/10.1109/ICMAP.2018.8354521

[9] Vavoulas, A., Sandalidis, H.G. and Varoutas, D. (2012) Weather Effects on FSO Network Connectivity. IEEE/OSA Journal of Optical Communications and Networking, 4, 734-740. https://doi.org/10.1364/JOCN.4.000734

[10] Nebuloni, R. and Capsoni, C. (2016) Effects of Adverse Weather on Free Space Optics. In: Uysal, M., et al., Eds., Optical Wireless Communications, Signals and Communication Technology, Springer International Publishing, Switzerland, 47-68.

[11] Das, S. and Chakraborty, M. (2016) ASK and PPM Modulation Based FSO System under Varying Weather Conditions. 2016 IEEE 7 th Annual Ubiquitous Computing, Electronics \& Mobile Communication Conference (UEMCON), New York, NY, 1-7. https://doi.org/10.1109/UEMCON.2016.7777825

[12] Kadhim, Dr.S.A., Shakir, A.A.J., Mohammad, Dr.A.N. and Mohammad, N.F. (2015) System Design and Simulation Using (OptiSystem7.0) for Performance Characterization of the Free Space Optical Communication System. International Journal of Innovative Research in Science, Engineering and Technology, 4, No. 6.

[13] Jain, D. and Mehra, R. (2017) Performance Analysis of Free Space Optical Communication System for S, C and L Band. International Conference on Computer, Communications and Electronics (Comptelix), Jaipur, 1-2 July 2017, 183-189.

[14] Shaina and Gupta, A. (2016) Comparative Analysis of Free Space Optical Communication System for Various Optical Transmission Windows under Adverse Weather Conditions. Twelfth International Multi-Conference on Information Processing, 89, 99-106. 